\title{
Approximation properties of $(p, q)$-Bernstein type operators
}

\author{
Zoltán Finta \\ Department of Mathematics, \\ Babeş-Bolyai University, Romania \\ email: fzoltan@math.ubbcluj.ro
}

\begin{abstract}
We introduce a new generalization of the q-Bernstein operators involving $(p, q)$-integers, and we establish some direct approximation results. Further, we define the limit $(p, q)$-Bernstein operator, and we obtain its estimation for the rate of convergence. Finally, we introduce the $(p, q)$-Kantorovich type operators, and we give a quantitative estimation.
\end{abstract}

\section{Introduction}

The applications of q-calculus in the field of approximation theory have led to the discovery of new generalizations of the Bernstein operators. The first generalization involving q-integers was obtained by Lupaş [7] in 1987. Ten years later Phillips [12] gave another generalization of the Bernstein operators introducing the so-called q-Bernstein operators. In comparison with Phillips' generalization, the Lupaş' generalization gives rational functions rather than polynomials. Nowadays, q-Bernstein operators form an area of an intensive research. A survey of the obtained results and references in this area during the first decade of study can be found in [11]. After that several well-known positive linear operators and other new operators have been generalized to their q-variants, and their approximation behavior have been studied (see e.g. [1] and [3]).

2010 Mathematics Subject Classification: 41A25, 41A36

Key words and phrases: $(p, q)$-integers, $(p, q)$-Bernstein operators, limit $(p, q)$-Bernstein operator, ( $p, q)$-Kantorovich operators, rate of convergence, modulus of continuity 
The $(p, q)$-calculus is a further new generalization of the $q$-calculus, its basic definitions and some properties may be found in the papers [6], [13], [14], [15]. The $(p, q)$-integers $[n]_{p, q}$ are defined by

$$
[n]_{p, q}=\frac{p^{n}-q^{n}}{p-q},
$$

where $\mathrm{n}=0,1,2, \ldots$ and $0<\mathrm{q}<\mathrm{p} \leq 1$. For $\mathrm{p}=1$, we recover the well-known q-integers (see [5]). Obviously

$$
[n]_{p, q}=p^{n-1}[n]_{q / p} .
$$

The $(p, q)$-factorials $[n]_{p, q}$ ! are defined by

$$
[n]_{p, q} !=\left\{\begin{aligned}
{[1]_{p, q}[2]_{p, q} \ldots[n]_{p, q}, } & \text { if } n \geq 1 \\
1, & \text { if } n=0
\end{aligned}\right.
$$

and the $(p, q)$-binomial coefficients are given by

$$
\left[\begin{array}{l}
n \\
k
\end{array}\right]_{p, q}=\frac{[n]_{p, q} !}{[k]_{p, q} ![n-k]_{p, q} !}, \quad 0 \leq k \leq n .
$$

Further, we set

$$
(a-b)_{p, q}^{n}=\left\{\begin{array}{rr}
(a-b)(p a-q b) \ldots\left(p^{n-1} a-q^{n-1} b\right), & \text { if } n \geq 1 \\
1, & \text { if } n=0 .
\end{array}\right.
$$

By simple computations, using (1), we get

$$
\begin{gathered}
{[n]_{\mathfrak{p}, q} !=p^{\mathfrak{n}(n-1) / 2}[n]_{\mathfrak{q} / \mathrm{p}} !} \\
{\left[\begin{array}{l}
n \\
k
\end{array}\right]_{p, q}=p^{\{n(n-1)-k(k-1)-(n-k)(n-k-1)\} / 2}\left[\begin{array}{l}
n \\
k
\end{array}\right]_{q / p}}
\end{gathered}
$$

and

$$
(a-b)_{p, q}^{n}=p^{n(n-1) / 2}(a-b)_{q / p}^{n},
$$

where

$$
(a-b)_{q}^{n}=\left\{\begin{array}{rr}
(a-b)(a-q b) \ldots\left(a-q^{n-1} b\right), & \text { if } n \geq 1 \\
1, & \text { if } n=0
\end{array}\right.
$$


in the case when $0<\mathrm{q}<1$.

The goal of the paper is to introduce a new generalization of the q-Bernstein operators involving $(p, q)$-integers. These $(p, q)$-Bernstein operators approximate each continuous function uniformly on $[0,1]$, and some direct approximation results are established with the aid of the modulus of continuity given by

$$
\omega(f ; \delta)=\sup \{|f(x)-f(y)|: x, y \in[0,1],|x-y| \leq \delta\}, \quad \delta>0,
$$

where $f \in C[0,1]$. Further, we define the limit $(p, q)$-Bernstein operator and we estimate the rate of convergence by the modulus of continuiuty (5). The concept of limit q-Bernstein operator was introduced by Il'inskii and Ostrovska [4], and its rate of convergence was established by Wang and Meng in [16]. Finally, we define a $(p, q)$-Kantorovich variant of the $(p, q)$-Bernstein operators, and we give a quantitative estimation using (5).

\section{2 ( $p, q)$-Bernstein operators}

For $0<q<p \leq 1, f \in C[0,1], x \in[0,1]$ and $n=1,2, \ldots$, we define the $(p, q)$-Bernstein polynomials as follows:

$$
B_{n, p, q}(f ; x)=\sum_{k=0}^{n} p^{\{k(k-1)-n(n-1)\} / 2}\left[\begin{array}{l}
n \\
k
\end{array}\right]_{p, q} x^{k}(1-x)_{p, q}^{n-k} f\left(p^{n} \frac{[k]_{p, q}}{[n]_{p, q}}\right) .
$$

For $\mathrm{p}=1$ and $0<\mathrm{q}<1$, we recover the $\mathrm{q}$-Bernstein polynomials (see [12]):

$$
B_{n, q}(f ; x)=\sum_{k=0}^{n}\left[\begin{array}{l}
n \\
k
\end{array}\right]_{q} x^{k}(1-x)_{q}^{n-k_{f}}\left(\frac{[k]_{q}}{[n]_{q}}\right) .
$$

Theorem 1 If the sequences $\left(\mathrm{p}_{\mathrm{n}}\right)$ and $\left(\mathrm{q}_{\mathrm{n}}\right)$ satisfy $0<\mathrm{q}_{\mathrm{n}}<\mathrm{p}_{\mathrm{n}} \leq 1$ for $\mathrm{n}=1,2, \ldots$, and $\mathrm{p}_{\mathrm{n}} \rightarrow 1, \mathrm{q}_{\mathrm{n}} \rightarrow 1, \mathrm{p}_{\mathrm{n}}^{\mathrm{n}} \rightarrow 1$ as $\mathrm{n} \rightarrow \infty$, then

$$
\left|B_{n, p_{n}, q_{n}}(f ; x)-f(x)\right| \leq 2 \omega\left(f ;\left(2\left(1-p_{n}^{n}\right) x^{2}+\frac{x(1-x)}{[n]_{q_{n} / p_{n}}}\right)^{1 / 2}\right)
$$

for all $\mathrm{f} \in \mathrm{C}[0,1]$ and $\mathrm{x} \in[0,1]$.

Proof. By (6), (3)-(4) and (1), we have

$$
B_{n, p, q}(f ; x)=\sum_{k=0}^{n}\left[\begin{array}{l}
n \\
k
\end{array}\right]_{q / p} x^{k}(1-x)_{q / p}^{n-k} f\left(p^{k} \frac{[k]_{q / p}}{[n]_{q / p}}\right) .
$$


Hence, in view of $[12,(13)]$, we obtain

$$
\mathrm{B}_{\mathfrak{n}, \mathfrak{p}, q}(1 ; x)=B_{n, q} / \mathfrak{p}(1 ; x)=1 .
$$

By (8) and $[12,(14)]$, we get

$$
p^{n} x=p^{n} B_{n, q / p}(t ; x) \leq B_{n, p, q}(t ; x)=\sum_{k=0}^{n}\left[\begin{array}{l}
n \\
k
\end{array}\right]_{q / p} x^{k}(1-x)_{q / p}^{n-k} p^{k} \frac{[k]_{q / p}}{[n]_{q / p}} .
$$

Analogously, by (8) and [12, (15)], we get

$$
\begin{aligned}
B_{n, p, q}\left(t^{2} ; x\right) & =\sum_{k=0}^{n}\left[\begin{array}{l}
n \\
k
\end{array}\right]_{q / p} x^{k}(1-x)_{q / p}^{n-k} p^{2 k} \frac{[k]_{q / p}^{2}}{[n]_{q / p}^{2}} \\
& \leq B_{n, q / p}\left(t^{2} ; x\right)=x^{2}+\frac{x(1-x)}{[n]_{q / p}} .
\end{aligned}
$$

On the other hand, it is known for (5) that

$$
\omega(f ; \lambda \delta) \leq(1+\lambda) \omega(f ; \delta),
$$

where $\lambda \geq 0$ and $\delta>0$. Then, by (8), [12, (13)], Hölder's inequality and (9)-(11), we obtain

$$
\begin{aligned}
\left|B_{n, p_{n}, q_{n}}(f ; x)-f(x)\right| & \leq \sum_{k=0}^{n}\left[\begin{array}{l}
n \\
k
\end{array}\right]_{q_{n} / p_{n}} x^{k}(1-x)_{q_{n} / p_{n}}^{n-k}\left|f\left(p_{n}^{k} \frac{[k]_{q_{n} / p_{n}}}{[n]_{q_{n} / p_{n}}}\right)-f(x)\right| \\
\leq & \sum_{k=0}^{n}\left[\begin{array}{l}
n \\
k
\end{array}\right]_{q_{n} / p_{n}} x^{k}(1-x)_{q_{n} / p_{n}}^{n-k} \omega\left(f ;\left|p_{n}^{k} \frac{[k]_{q_{n} / p_{n}}}{[n]_{q_{n} / p_{n}}}-x\right|\right) \\
\leq & \omega(f ; \delta) \sum_{k=0}^{n}\left[\begin{array}{c}
n \\
k
\end{array}\right]_{q_{n} / p_{n}} x^{k}(1-x)_{q_{n} / p_{n}}^{n-k}\left(1+\delta^{-1}\left|p_{n}^{k} \frac{[k]_{q_{n} / p_{n}}}{[n]_{q_{n} / p_{n}}}-x\right|\right) \\
\leq & \omega(f ; \delta)\left\{1+\delta^{-1}\left(\sum_{k=0}^{n}\left[\begin{array}{c}
n \\
k
\end{array}\right]_{q_{n} / p_{n}} x^{k}(1-x)_{q_{n} / p_{n}}^{n-k}\right.\right. \\
& \left.\left.\times\left(p_{n}^{k} \frac{[k]_{q_{n} / p_{n}}}{[n]_{q_{n} / p_{n}}}-x\right)^{2}\right)^{1 / 2}\right\} \\
= & \omega(f ; \delta)\left\{1+\delta^{-1}\left(B_{n, p_{n}, q_{n}}\left(t^{2} ; x\right)-2 x B_{n, p_{n}, q_{n}}(t ; x)\right.\right. \\
& \left.\left.+x^{2} B_{n, p_{n}, q_{n}}(1 ; x)\right)^{1 / 2}\right\}
\end{aligned}
$$




$$
\begin{aligned}
& \leq \omega(f ; \delta)\left\{1+\delta^{-1}\left(x^{2}+\frac{x(1-x)}{[n]_{\mathfrak{q}_{n} / p_{n}}}-2 p_{n}^{n} x^{2}+x^{2}\right)^{1 / 2}\right\} \\
& =\omega(f ; \delta)\left\{1+\delta^{-1}\left(2\left(1-p_{n}^{n}\right) x^{2}+\frac{x(1-x)}{[n]_{q_{n} / p_{n}}}\right)^{1 / 2}\right\} .
\end{aligned}
$$

Choosing $\delta=\left(2\left(1-p_{n}^{n}\right) x^{2}+\frac{x(1-x)}{[n]_{q_{n} / p_{n}}}\right)^{1 / 2}$, we arrive at the statement of our theorem.

Theorem 2 If the sequences $\left(\mathrm{p}_{\mathrm{n}}\right)$ and $\left(\mathrm{q}_{\mathrm{n}}\right)$ satisfy $0<\mathrm{q}_{\mathrm{n}}<\mathrm{p}_{\mathrm{n}} \leq 1$ for $\mathrm{n}=1,2, \ldots$, and $\mathrm{p}_{\mathrm{n}} \rightarrow 1, \mathrm{q}_{\mathrm{n}} \rightarrow 1, \mathrm{p}_{\mathrm{n}}^{\mathrm{n}} \rightarrow 1$ as $\mathrm{n} \rightarrow \infty$, then

$$
\left|B_{n, p_{n}, q_{n}}(f ; x)-B_{n, q_{n} / p_{n}}(f ; x)\right| \leq \omega\left(f ; 1-p_{n}^{n}\right)
$$

for all $\mathrm{f} \in \mathrm{C}[0,1]$ and $\mathrm{x} \in[0,1]$.

Proof. Because $\left|p^{k} \frac{[k]_{q / p}}{[n]_{q / p}}-\frac{[k]_{q / p}}{[n]_{q / p}}\right| \leq 1-p^{k} \leq 1-p^{n}$ for $k=0,1, \ldots, n$, we find from (8), (7) and $[12,(13)]$, that

$$
\begin{aligned}
& \left|B_{n, p_{n}, q_{n}}(f ; x)-B_{n, q_{n} / p_{n}}(f ; x)\right| \\
& \leq \sum_{k=0}^{n}\left[\begin{array}{c}
n \\
k
\end{array}\right]_{q_{n} / p_{n}} x^{k}(1-x)_{q_{n} / p_{n}}^{n-k}\left|f\left(p_{n}^{k} \frac{[k]_{q_{n} / p_{n}}}{[n]_{q_{n} / p_{n}}}\right)-f\left(\frac{[k]_{q_{n} / p_{n}}}{[n]_{q_{n} / p_{n}}}\right)\right| \\
& \leq \sum_{k=0}^{n}\left[\begin{array}{c}
n \\
k
\end{array}\right]_{q_{n} / p_{n}} x^{k}(1-x)_{q_{n} / p_{n}}^{n-k} \omega\left(f ;\left|p_{n}^{k} \frac{[k]_{q_{n} / p_{n}}}{[n]_{q_{n} / p_{n}}}-\frac{[k]_{q_{n} / p_{n}}}{[n]_{q_{n} / p_{n}}}\right|\right) \\
& \leq \omega\left(f ; 1-p_{n}^{n}\right) B_{n, q_{n} / p_{n}}(1 ; x)=\omega\left(f ; 1-p_{n}^{n}\right),
\end{aligned}
$$

which is the required estimation.

Remark 1 There exist sequences $\left(p_{n}\right)$ and $\left(q_{n}\right)$ with the properties enumerated in Theorem 1: $p_{n}=1-\frac{1}{(n+1)^{2}}$ and $q_{n}=1-\frac{1}{n+1}, n=1,2, \ldots$

We also mention, if $0<\mathrm{q}_{\mathrm{n}}<\mathrm{p}_{\mathrm{n}} \leq 1$ for $\mathrm{n}=1,2, \ldots, \mathrm{p}_{\mathrm{n}} \rightarrow 1$ and $\mathrm{q}_{\mathrm{n}} \rightarrow 1$ as $\mathrm{n} \rightarrow \infty$, then $[\mathrm{n}]_{\mathfrak{q}_{\mathrm{n}} / \mathrm{p}_{\mathfrak{n}}} \rightarrow \infty$ and $\frac{[\mathrm{n}]_{\mathrm{q}_{\mathfrak{n}} / \mathrm{p}_{\mathfrak{n}}}}{[\mathrm{n}+1]_{\mathrm{q}_{\mathrm{n}} / \mathrm{p}_{\mathfrak{n}}}} \rightarrow 1$ as $\mathrm{n} \rightarrow \infty$.

Remark 2 In [9] and [10] are introduced two different generalizations of the $\mathrm{q}$-Bernstein polynomials $(7)$ involving $(\mathrm{p}, \mathrm{q})$-integers. The first one does not preserve even the constant functions, and the second one is a $(q / p)$-Bernstein polynomial. Our ( $p, q)$-Bernstein polynomials defined by (6) are different from the above mentioned generalizations. The advantage of (6) is that it allows us to introduce the limit ( $p, q)$-Bernstein operator. 


\section{Limit ( $p, q)$-Bernstein operator}

For $q \in(0,1)$, Il'inskii and Ostrovska proved in [4] that for each $f \in C[0,1]$, the sequence $\left(B_{n, q}(f ; x)\right)$ converges to $B_{\infty, q}(f ; x)$ as $n \rightarrow \infty$ uniformly for $x \in[0,1]$, where

$$
B_{\infty, q}(f ; x)=\left\{\begin{array}{rr}
\sum_{k=0}^{\infty} f\left(1-q^{k}\right) \frac{x^{k}}{(1-q)^{k}[k]_{q} !} \prod_{s=0}^{\infty}\left(1-q^{s} x\right), & \text { if } 0 \leq x<1 \\
f(1), & \text { if } x=1
\end{array}\right.
$$

is the limit q-Bernstein operator. Wang and Meng [16] proved for all $f \in C[0,1]$ and $x \in[0,1]$ that

$$
\left|B_{n, q}(f ; x)-B_{\infty, q}(f ; x)\right| \leq\left(2+\frac{4}{q(1-q)} \ln \frac{1}{1-q}\right) \omega\left(f ; q^{n}\right) .
$$

For $0<\mathrm{q}<\mathrm{p} \leq 1$, the limit $(\mathrm{p}, \mathrm{q})$-Bernstein operator $\mathrm{B}_{\infty, \mathrm{p}, \mathrm{q}}: \mathrm{C}[0,1] \rightarrow$ $\mathrm{C}[0,1]$ is defined as follows:

$$
B_{\infty, p, q}(f ; x)=\left\{\begin{array}{rr}
\sum_{k=0}^{\infty} f\left(p^{k}-q^{k}\right) \frac{p^{(k+1) k / 2} \chi^{k}}{(p-q)^{k}[k]_{p, q} !} \prod_{s=0}^{\infty} \frac{p^{s}-q^{s} x}{p^{s}}, & \text { if } x \in[0,1) \\
f(1), & \text { if } x=1 .
\end{array}\right.
$$

Theorem 3 Let $\mathrm{p}, \mathrm{q} \in(0,1)$ be given such that $\mathrm{p}^{2}<\mathrm{q}<\mathrm{p}$. Then, for every $\mathrm{f} \in \mathrm{C}[0,1], \mathrm{x} \in[0,1]$ and $\mathrm{n}=1,2, \ldots$, we have

$$
\left|B_{n, p, q}(f ; x)-B_{\infty, p, q}(f ; x)\right| \leq\left(4+\frac{6 p^{2}}{q(p-q)} \ln \frac{p}{p-q}\right) \omega\left(f ;\left(\frac{q}{p}\right)^{n}\right) .
$$

Proof. Due to (13) and (2), we have

$$
B_{\infty, p, q}(f ; x)=\sum_{k=0}^{\infty} f\left(p^{k}-q^{k}\right) \frac{x^{k}}{\left(1-\frac{q}{p}\right)^{k}[k]_{q / p} !} \prod_{s=0}^{\infty}\left(1-\left(\frac{q}{p}\right)^{s} x\right) .
$$

We set

$w_{n, k}(\mathrm{q} ; x)=\left[\begin{array}{l}\mathrm{n} \\ \mathrm{k}\end{array}\right]_{\mathrm{q}} x^{\mathrm{k}}(1-x)_{\mathrm{q}}^{\mathrm{n}-\mathrm{k}}$ and $w_{\infty, \mathrm{k}}(\mathrm{q} ; \mathrm{x})=\frac{x^{\mathrm{k}}}{(1-\mathrm{q})^{\mathrm{k}}[\mathrm{k}]_{\mathrm{q}} !} \prod_{s=0}^{\infty}\left(1-\mathrm{q}^{\mathrm{s}} \mathrm{x}\right)$. 
Then, in view of (9) and [16, p. 154, (2.3)], we obtain

$$
\sum_{k=0}^{n} w_{n, k}\left(\frac{q}{p} ; x\right)=\sum_{k=0}^{\infty} w_{\infty, k}\left(\frac{q}{p} ; x\right)=1 \text {. }
$$

Using (8), (14) and (15), we find

$$
\begin{aligned}
\left|B_{n, p, q}(f ; x)-B_{\infty, p, q}(f ; x)\right| & \mid \sum_{k=0}^{n} w_{n, k}\left(\frac{q}{p} ; x\right)\left\{f\left(p^{k} \frac{[k]_{q / p}}{[n]_{q / p}}\right)-f\left(p^{k}-q^{k}\right)\right\} \\
\quad+ & \sum_{k=0}^{n}\left\{w_{n, k}\left(\frac{q}{p} ; x\right)-w_{\infty, k}\left(\frac{q}{p} ; x\right)\right\}\left\{f\left(p^{k}-q^{k}\right)-f\left(p^{n}\right)\right\} \\
& -\sum_{k=n+1}^{\infty} w_{\infty, k}\left(\frac{q}{p} ; x\right)\left\{f\left(p^{k}-q^{k}\right)-f\left(p^{n}\right)\right\} \mid \\
\leq & \sum_{k=0}^{n} w_{n, k}\left(\frac{q}{p} ; x\right)\left|f\left(p^{k} \frac{[k]_{q / p}}{[n]_{q} / p}\right)-f\left(p^{k}-q^{k}\right)\right| \\
& +\sum_{k=0}^{n}\left|w_{n, k}\left(\frac{q}{p} ; x\right)-w_{\infty, k}\left(\frac{q}{p} ; x\right)\right|\left|f\left(p^{k}-q^{k}\right)-f\left(p^{n}\right)\right| \\
& +\sum_{k=n+1}^{\infty} w_{\infty, k}\left(\frac{q}{p} ; x\right)\left|f\left(p^{k}-q^{k}\right)-f\left(p^{n}\right)\right| \\
=: & I_{1}+I_{2}+I_{3} .
\end{aligned}
$$

The estimation of $\mathrm{I}_{1}$ : by (1), we have

$$
\begin{aligned}
& \left|p^{k} \frac{[k]_{q / p}}{[n]_{q / p}}-\left(p^{k}-q^{k}\right)\right|=\frac{[k]_{q / p}}{[n]_{q / p}}\left|p^{k}-\left(p^{k}-q^{k}\right) p^{k-n} \frac{[n]_{p, q}}{[k]_{p, q}}\right| \\
& \quad \leq\left|p^{k}-\left(p^{k}-q^{k}\right) p^{k-n} \frac{p^{n}-q^{n}}{p^{k}-q^{k}}\right|=p^{k}\left(\frac{q}{p}\right)^{n} \leq\left(\frac{q}{p}\right)^{n}
\end{aligned}
$$

for $k=0,1, \ldots, n$. Hence, by (15),

$$
I_{1} \leq \sum_{k=0}^{n} w_{n, k}\left(\frac{q}{p} ; x\right) \omega\left(f ;\left|p^{k} \frac{[k]_{q / p}}{[n]_{q / p}}-\left(p^{k}-q^{k}\right)\right|\right) \leq \omega\left(f ;\left(\frac{q}{p}\right)^{n}\right) .
$$


The estimation of $\mathrm{I}_{2}$ : for $\mathrm{k}=0,1, \ldots, \mathrm{n}$, we have $\left|\mathrm{p}^{\mathrm{k}}-\mathrm{q}^{\mathrm{k}}-\mathrm{p}^{\mathrm{n}}\right| \leq \mathrm{p}^{\mathrm{k}}(1-$ $\left.\mathrm{p}^{\mathrm{n}-\mathrm{k}}\right)+\mathrm{q}^{\mathrm{k}} \leq \mathrm{p}^{\mathrm{k}}+\mathrm{q}^{\mathrm{k}}$. Hence, by $(12)$,

$$
\begin{aligned}
& \left|f\left(p^{k}-q^{k}\right)-f\left(p^{n}\right)\right| \leq \omega\left(f ;\left|p^{k}-q^{k}-p^{n}\right|\right) \leq \omega\left(f ; p^{k}+q^{k}\right) \\
& \quad=\omega\left(f ; \frac{p^{k}+q^{k}}{(q / p)^{n}}\left(\frac{q}{p}\right)^{n}\right) \leq\left(1+\frac{p^{k}+q^{k}}{(q / p)^{n}}\right) \omega\left(f ;\left(\frac{q}{p}\right)^{n}\right) .
\end{aligned}
$$

But

$$
\begin{aligned}
& \left(1+\frac{p^{k}+q^{k}}{(q / p)^{n}}\right)\left(\frac{p}{q}\right)^{k}=\left(\frac{q}{p}\right)^{-n}\left(\left(\frac{q}{p}\right)^{n}+p^{k}+q^{k}\right)\left(\frac{p}{q}\right)^{k} \\
& =\left(\frac{q}{p}\right)^{-n}\left(\left(\frac{q}{p}\right)^{n-k}+\left(\frac{p^{2}}{q}\right)^{k}+p^{k}\right) \leq 3\left(\frac{q}{p}\right)^{-n},
\end{aligned}
$$

because $p^{2}<q<p$ and $k=0,1, \ldots, n$. Then, by (18), we obtain

$$
\begin{aligned}
I_{2} & \leq \sum_{k=0}^{n}\left|w_{n, k}\left(\frac{\mathrm{q}}{\mathrm{p}} ; x\right)-w_{\infty, k}\left(\frac{\mathrm{q}}{\mathrm{p}} ; x\right)\right| 3\left(\frac{\mathrm{q}}{\mathrm{p}}\right)^{\mathrm{k}-\mathrm{n}} \omega\left(\mathrm{f} ;\left(\frac{\mathrm{q}}{\mathrm{p}}\right)^{\mathrm{n}}\right) \\
& =3\left(\frac{\mathrm{q}}{\mathrm{p}}\right)^{-\mathrm{n}} \omega\left(\mathrm{f} ;\left(\frac{\mathrm{q}}{\mathrm{p}}\right)^{\mathrm{n}}\right) \sum_{\mathrm{k}=0}^{n}\left(\frac{\mathrm{q}}{\mathrm{p}}\right)^{\mathrm{k}}\left|w_{n, k}\left(\frac{\mathrm{q}}{\mathrm{p}} ; x\right)-w_{\infty, k}\left(\frac{\mathrm{q}}{\mathrm{p}} ; x\right)\right| .
\end{aligned}
$$

Taking into account the estimation

$$
\sum_{k=0}^{n} q^{k}\left|w_{n, k}(q ; x)-w_{\infty, k}(q ; x)\right| \leq \frac{2 q^{n}}{q(1-q)} \ln \frac{1}{1-q},
$$

where $0<\mathrm{q}<1$ (see $[16$, p. $156,(2.9)]$ ), we find that

$$
I_{2} \leq \frac{6 p^{2}}{q(p-q)} \ln \frac{p}{p-q} \omega\left(f ;\left(\frac{q}{p}\right)^{n}\right) .
$$

The estimation of $I_{3}$ : for $k \geq n+1$, we have $\left|p^{k}-q^{k}-p^{n}\right| \leq p^{n}\left(1-p^{k-n}\right)+q^{k} \leq$ $\mathrm{p}^{\mathrm{n}}+\mathrm{q}^{\mathrm{n}}$. Hence, by (12) and $\mathrm{p}^{2}<\mathrm{q}<\mathrm{p}$, we get

$$
\begin{aligned}
& \left|f\left(p^{k}-q^{k}\right)-f\left(p^{n}\right)\right| \leq \omega\left(f ;\left|p^{k}-q^{k}-p^{n}\right|\right) \leq \omega\left(f ; p^{n}+q^{n}\right) \\
& \quad \leq\left(1+\frac{p^{n}+q^{n}}{(q / p)^{n}}\right) \omega\left(f ;\left(\frac{q}{p}\right)^{n}\right)=\left(1+\left(\frac{p^{2}}{q}\right)^{n}+p^{n}\right) \omega\left(f ;\left(\frac{q}{p}\right)^{n}\right) \\
& \quad \leq 3 \omega\left(f ;\left(\frac{q}{p}\right)^{n}\right) .
\end{aligned}
$$


Then, by (15),

$$
I_{3} \leq 3 \omega\left(f ;\left(\frac{q}{p}\right)^{n}\right) \sum_{k=n+1}^{\infty} w_{\infty, k}\left(\frac{q}{p} ; x\right) \leq 3 \omega\left(f ;\left(\frac{q}{p}\right)^{n}\right) .
$$

Combining (16)-(17) and (19)-(20), we obtain the statement of the theorem.

\section{4 ( $p, q)$-Kantorovich operators}

$\operatorname{Our}(p, q)$-Kantorovich operators are defined as follows:

$$
\begin{aligned}
K_{n, p, q}(f ; x)= & \frac{[n+1]_{p, q}}{p^{n}} \sum_{k=0}^{n} p^{\{k(k-1)-n(n-1)\} / 2}\left[\begin{array}{l}
n \\
k
\end{array}\right]_{p, q} x^{k}(1-x)_{p, q}^{n-k} \\
& \times q^{-k} \int_{p^{n+1} \frac{[k] p, q}{[n+1]_{p}, q}}^{p^{n} \frac{k k+1]_{p}, q}{[n+1)}} f(u) d_{q / p}^{R} u
\end{aligned}
$$

where $f \in C[0,1], x \in[0,1], n=1,2, \ldots$, and the Riemann type q-integral of $\mathrm{f}$ over the interval $[\mathrm{a}, \mathrm{b}](0 \leq \mathrm{a}<\mathrm{b} ; 0<\mathrm{q}<1)$ is given by (see [2], [8])

$$
\int_{a}^{b} f(u) d_{q}^{R} u=(1-q)(b-a) \sum_{j=0}^{\infty} q^{j} f\left(a+(b-a) q^{j}\right) .
$$

Remark 3 In $[15]$ the ( $p, q)$-integral of $f$ over the interval $[0, a]$ is defined as

$$
\int_{0}^{a} f(u) d_{p, q} u=(p-q) a \sum_{j=0}^{\infty} \frac{q^{j}}{p^{j+1}} f\left(a \frac{q^{j}}{p^{j+1}}\right),
$$

where $0<\mathrm{q}<\mathrm{p} \leq 1$. But $\frac{1}{\mathrm{p}} \mathrm{a} \notin[0, \mathrm{a}]$ for $0<\mathrm{p}<1$ (in the sum the case $j=0$ ), thus the function $f$ is not defined at $\frac{1}{p} a$. For this reason we use the Riemann type (q/p)-integral in (21).

Theorem 4 If the sequences $\left(\mathrm{p}_{\mathrm{n}}\right)$ and $\left(\mathrm{q}_{\mathrm{n}}\right)$ satisfy $0<\mathrm{q}_{\mathrm{n}}<\mathrm{p}_{\mathrm{n}} \leq 1$ for $\mathrm{n}=1,2, \ldots$, and $\mathrm{p}_{\mathrm{n}} \rightarrow 1, \mathrm{q}_{\mathrm{n}} \rightarrow 1, \mathrm{p}_{\mathrm{n}}^{\mathrm{n}} \rightarrow 1$ as $\mathrm{n} \rightarrow \infty$, then

$$
\left|K_{n, p_{n}, q_{n}}(f ; x)-f(x)\right| \leq 2 \omega\left(f ; \sqrt{\delta_{n}(x)}\right)
$$


for all $\mathrm{f} \in \mathrm{C}[0,1]$ and $\mathrm{x} \in[0,1]$, where

$$
\begin{aligned}
\delta_{n}(x)= & \left\{2\left(1-p_{n}^{n}\right) \frac{[n]_{\mathfrak{q}_{n} / p_{n}}}{[n+1]_{\mathfrak{q}_{n} / p_{n}}}+\left(1-\frac{[n]_{\mathfrak{q}_{n} / p_{n}}}{[n+1]_{\mathfrak{q}_{n} / p_{n}}}\right)^{2}-\frac{[n]_{\mathfrak{q}_{n} / p_{n}}}{[n+1]_{\mathfrak{q}_{n} / p_{n}}^{2}}\right\} \\
& \times x^{2}+3 \frac{[n]_{\mathfrak{q}_{n} / p_{n}}}{[n+1]_{\mathfrak{q}_{n} / p_{n}}} x+\frac{1}{[n+1]_{\mathfrak{q}_{n} / p_{n}}^{2}} .
\end{aligned}
$$

Proof. By (21), (3)-(4) and (1), we have

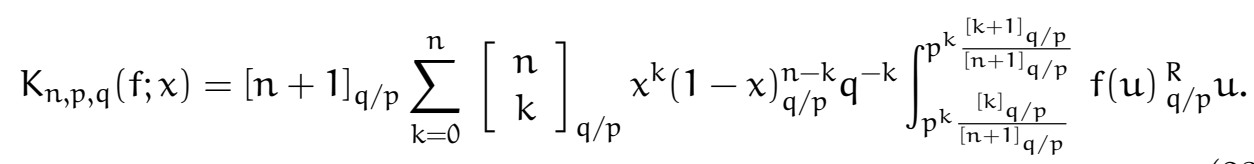

By simple computations, using (22), we obtain

$$
\begin{gathered}
\int_{p^{k}}^{p^{k} \frac{[k+1]_{q / p}}{[n+1]_{q} / p}} 1 d_{q / p}^{R} u=p^{k} \frac{[k+1]_{q / p}-[k]_{q / p}}{[n+1]_{q / p}}=\frac{q^{k}}{[n+1]_{q / p}}, \\
\int_{p^{k} \frac{[k]}{[n+1]_{q} / p}}^{p^{k} \frac{[k+1]_{q} / p}{[n+1]}} u d_{q / p}^{R} u=\frac{q^{k}}{[n+1]_{q / p}}\left(p^{k} \frac{[k]_{q / p}}{[n+1]_{q / p}}+\frac{p}{p+q} \frac{q^{k}}{[n+1]_{q / p}}\right)
\end{gathered}
$$

and

$$
\begin{aligned}
\int_{p^{k} \frac{[k]_{q} / p}{[n+1]_{q} / p}}^{p^{k} \frac{[k+1]_{q} / p}{\left[n+1 q_{p}\right.}} u^{2} d_{q / p}^{R} u= & \frac{q^{k}}{[n+1]_{q / p}}\left(p^{2 k} \frac{[k]_{q / p}^{2}}{[n+1]_{q / p}^{2}}+\frac{2 p}{p+q} p^{k} \frac{[k]_{q / p}}{[n+1]_{q / p}}\right. \\
& \left.\times \frac{q^{k}}{[n+1]_{q / p}}+\frac{p^{2}}{p^{2}+p q+q^{2}} \frac{q^{2 k}}{[n+1]_{q / p}^{2}}\right) .
\end{aligned}
$$

In what follows, taking into account (23)-(26), the proof is similar to the proof of Theorem 1, therefore we omit the details.

\section{References}

[1] A. Aral, V. Gupta, R. P. Agarwal, Applications of q-Calculus in Operator Theory, Springer, New York, 2012.

[2] H. Gauchman, Integral inequalities in q-calculus, Comput. Math. Appl., 47 (2004), 281-300. 
[3] V. Gupta, R. P. Agarwal, Convergence Estimates in Approximation Theory, Springer, New York, 2014.

[4] A. Il'inskii, S. Ostrovska, Convergence of generalized Bernstein polynomials, J. Approx. Theory., 116 (1) (2002), 100-112.

[5] V. Kac, P. Cheung, Quantum Calculus, Springer, New York, 2002.

[6] K. Khan, D. K. Lobiyal, Bézier curves based on Lupaş (p, q)-analogue of Bernstein polynomials in CAGD, arXiv:1505.01810[cs.GR].

[7] A. Lupaş, A q-analogue of the Bernstein operator, Seminar on Numerical and Statistical Calculus, 9 (1987), 85-92.

[8] S. Marinković, P. Rajković, M. Stanković, The inequalities for some type of q-integrals, Comput. Math. Appl., 56 (2008), 2490-2498.

[9] M. Mursaleen, K. J. Ansari, A. Khan, On (p, q)-analogue of Bernstein operators, Appl. Math. Comput., 266 (2015), 874-882.

[10] M. Mursaleen, K. J. Ansari, A. Khan, Some approximation results for Bernstein-Kantorovich operators based on ( $p, q)$-calculus, arXiv:1504.05887v4[math.CA] 15 Jan 2016.

[11] S. Ostrovska, The first decade of the q-Bernstein polynomials: results and perspectives, J. Math. Anal. Approx. Theory, 2 (1) (2007), 35-51.

[12] G. M. Phillips, Bernstein polynomials based on the q-integers, Ann. Numer. Math., 4 (1997), 511-518.

[13] V. Sahai, S. Yadav, Representations of two parameter quantum algebras and p, q-special functions, J. Math. Anal. Appl., 335 (2007), 268-279.

[14] P. N. Sadjang, On the (p,q)-Gamma and the (p, q)-Beta functions, arXiv:1506.07394v1. 22 Jun 2015.

[15] P. N. Sadjang, On the fundamental theorem of $(p, q)$-calculus and some (p, q)-Taylor formulas, arXiv:1309.3934[math.QA] 22 Aug 2013.

[16] H. Wang, F. Meng, The rate of convergence of q-Bernstein polynomials for $0<\mathrm{q}<1$, J. Approx. Theory, 136 (2005), 151-158. 